\title{
Finger Vein Image Enhancement Algorithm Based on the Grayscale Morphology
}

\author{
Chenxuan \\ (Zhejiang Industry Polytechnic College Shaoxing Zhejiang 312000 China) \\ 734140999@qq.com \\ Zhangjun \\ (HANGZHOU DIANZI UNIVERSITY HANGZHOU Zhejiang 310018 China) \\ 12584623@qq.com \\ Youlin \\ (HANGZHOU DIANZI UNIVERSITY HANGZHOU Zhejiang 310018China) \\ 78451236@qq.com \\ Wangchangliang \\ (Zhejiang Industry Polytechnic College Shaoxing Zhejiang 312000 China) \\ 7452128@qqcom \\ Xufangheng \\ (Zhejiang Industry Polytechnic College Shaoxing Zhejiang 312000 China) \\ 45128962@qq.com

\section{Abstract}

As the information secturity technology constantly develops, finger vein feature technology has become a new technology. The paper proposes a Finger Vein Image Enhancement Algorithm Based on the Grayscale Morphology (FVGM). The algorithm first uses a group of stuctural elements with the four directions for respective dilation operation of the image, and gets the smoothed finger vein image by weighting method. Simulation results show tha NVGM algorithm can effectively improve the definition of intravenous ines in the finger vein image.

Keywords: Information Security; Finger Vein; Grayscale Morphology

\section{Introduction}

As the Internet technology continuously develops, how to ensure the personal information sécurity has become an urgent problem to be solved. The adoption of traditional identification has been unable to adapt to the requirements of modern information security, such as ID card, debit card or setting passwords (such as user name, account number, etc.) and password. In particular, the information retention means by these traditional methods are relatively original, and it is thus easy to cause issues, including loss of identity information. Therefore, the use of biometric features recognition for personal identity verification is the safest and most reliable requirement [1].

At present, the main practical biometric feature identification technology consists of fingerprint recognition, face recognition, palm print recognition, finger vein recognition and other recognition technologies [2-4]. Among them, the finger vein recognition is a novel recognition technology, which mainly identify by the vein hidden in the human finger pulp. The literature [5] proposed a personalized weighted fusion recognition method based on finger vein and finger contours. It first classified the samples in accordance with the original score, and then obtained the sample classification confidence score according to the classification results. Compared with the original score, the classification confidence score was added to the classification information, which can 
provide more effective information for later fusion. At last, in order to reflect the differences between individuals, this paper uses the individualized weights for fusion of classification confidence score and verifies the effectiveness of the method based on the experimental results on the self-built database. The literature [6] proposed a finger vein recognition method that fuses the local features and global features. It applied local binary pattern to extract local features of finger vein, used the Hamming distance to calculate the matching score; applied two-way two-dimensional principal component analysis method to extract global features of finger vein, used the Euclidean distance to calculate the matching score; fused the two matching scores on the basis of score grade to produce the recognition result. Experimental results show that local features and global features are well complemented, which have effectively improved the recognition accuracy. The literature [7] proposed a histogram equalization method based on homomorphic filtering cubic convolution interpolation. Based on the local histogram equalization treatment, it used the homomorphic filtering transform to treat each sub-block's low frequency information and re-determined the gray value through the image interpolation. Experiments show that this method can effectively improve the image contrast inadequacy and uneven illumination. The literature $[8]$ proposed a new finger vein recognition method, namely, taking the essential features of intravenous internal topology as the principle for matching the finger vein. It first extracts the endpoints and the intersection points over the thinning restored image, calculated the relative distance between the feature points, and finally completed the finger vein image recognition by comparing the distance value. The method combines the vein features, takes full advantage of the essential attribute of topology, unnecessary to position and easy. The experimental results show that this method quickly and accurately identities, overcomes the translation and rotation effects on the recognition results to a certain extent, and has practical values. The literature [9] proposed a linear discriminant analysis method directly based on sub-image vector, that is, modular PCA finger vein feature extraction algorithm. The above algorithm identifies and analyzes the obtained sub-images by segmenting the images and using PCA The use of the sub-image matrix can effectively extract the local image features, avoid the use of singular value decomposition theory, and the process is simple. Experimental results shoy that the proposed method outperforms the traditional PCA algorithm and wavelet moment fusion PCA method in terms of recognition performance and the recognition rate has been effectively improved. The literature [10] proposed a background innage-based structure to obtain the primary residual image, obtain the secondary residual Image by adjusting the scaling factor and obtain the vein features in an indirect manner. After comparing the algorithm with other direct extraction algorithms, the results indicate that the proposed algorithm is simple, easy to implement. In particutar, the obtained secondary residual image gets more reasonable feature information in terms of fuzzy and low-quality images than other algorithms, being featured by strong authenticity and the realization of accurate, continuous and smooth requirements.

Due to various limitations, the collected finger vein image is featured by poor quality and failure of feature extraction, which has affected the recognition rate to a certain extent. To solve these issues, this paper extracts the algorithm for the finger vein feature, and presents a Finger Vein Image Enhancement Based on the Grayscale Morphology (referred to as FVGM). Simulation results show that FVGM algorithm can effectively improve the quality of finger vein image.

\section{Finger Vein Image Imaging Principle}

When the infrared light illuminates the finger vein, the hemoglobin can absorb the infrared lights. Therefore, among the camera-captured images, the vein position is darker than other finger parts, so as to obtain finger vein images with certain texture features. 
Figure 1-1 is a schematic diagram of finger vein image capture device, and Figure 1-2 is the collected finger vein image sample.

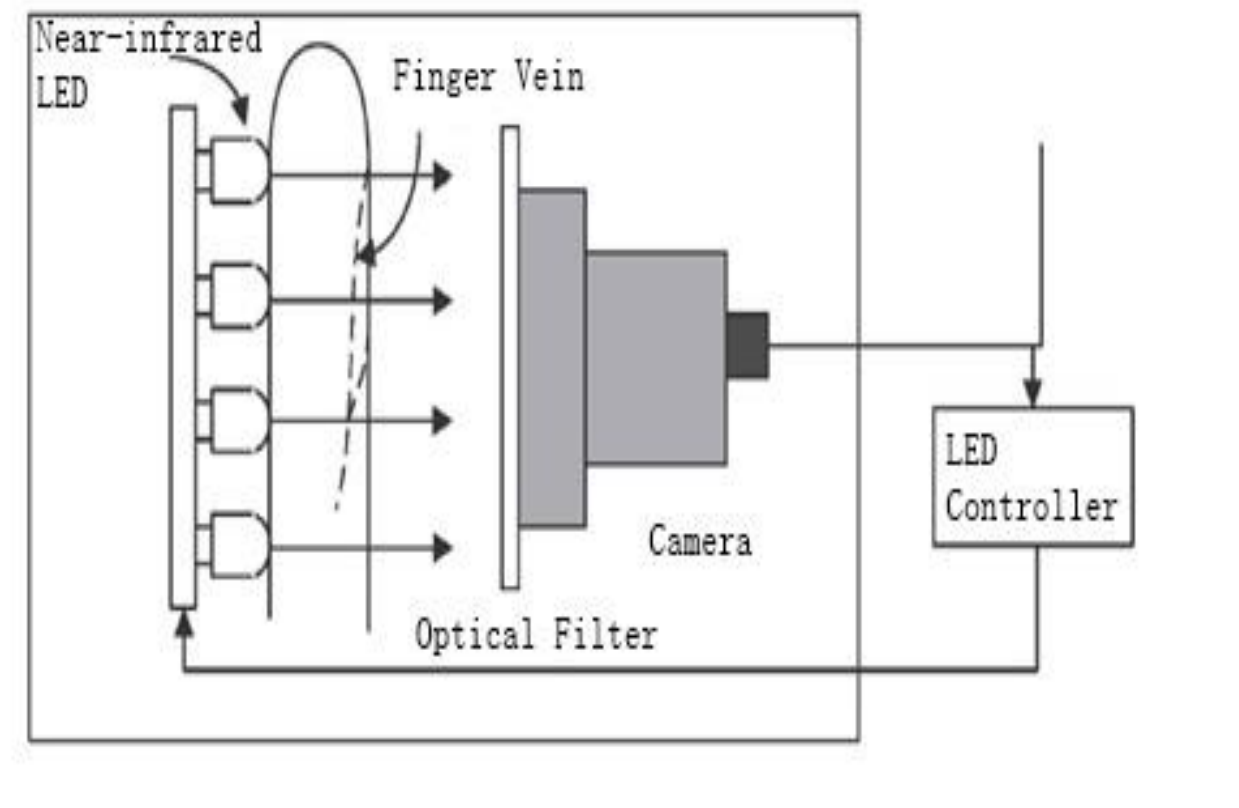

Figure 1. Schematic Diagram of Capture Device

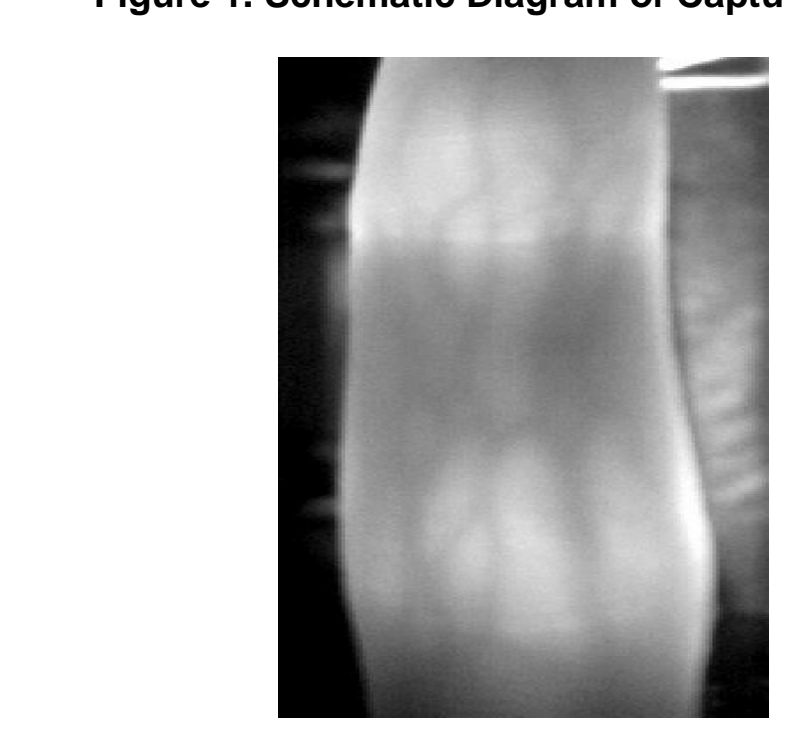

Figure 2. Schematic Diagram of Collected Finger Vein Image

\section{Finger Vein Image Enhancement Based on Grayscale Morphology}

\subsection{Construction of Morphology Filter}

The finger vein image usually has complex information, and the single structural element cannot effectively detect the image information, so the method of using different structural elements for image detection has been widely used. Among the finger vein images, the finger intravenous lines are characterized by directional and continuous property, and intravenous lines are mainly distributed along the direction of the finger, so the finger vein image can be smoothed by using multi-directional linear structure elements. This paper uses four $3 \times 3$ structural elements to construct the image 
smoothing filter, and the four structural elements are shown in Figure 3(a)-3(d).

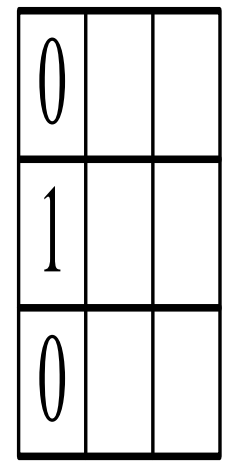

(a) $b 1$

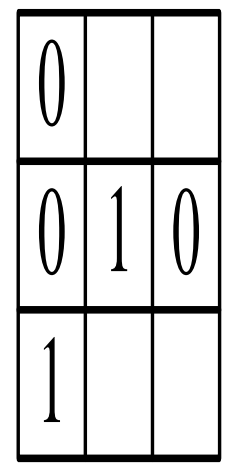

(b) $b 2$

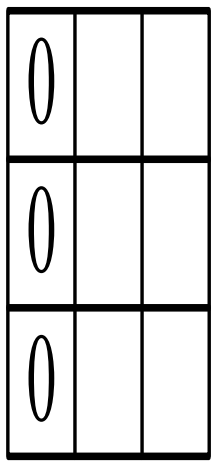

(c) $b 3$

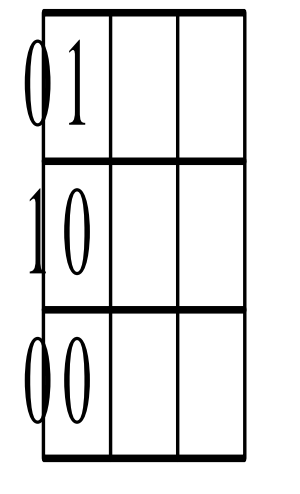

(d) $b^{4} \mathrm{C}$

Figure 3. Structural Elements in Four Directions

In Figure 3, $b 1, b 2, b 3$ and $b 4$ respectively sand for the structural elements in $0^{\circ}$ direction, $45^{\circ}$ direction, $90^{\circ}$ direction and $135^{\circ}$ direction. In grayscale morphological operation, the expansion operation will filter out the black areas smaller than the structural elements in the image, and the blackareas may be noise or may be vein. Besides, due to the vein's continuous and directional property, when the vein pattern information is filtered by the dilation operation in a eertain direction, the vein pattern in the other direction will be retained "Due to irregular distribution, noise will be filtered during dilation. The construction process of morphology filter is as follows:

(1) Carry out dilation operation by respectively using $b 1, b 2, b 3, b 4$ (Formula (1) - Formula (4)) and get the four sub-images $f_{b 1}, f_{b 2}, f_{b 3}, f_{b 4}$.

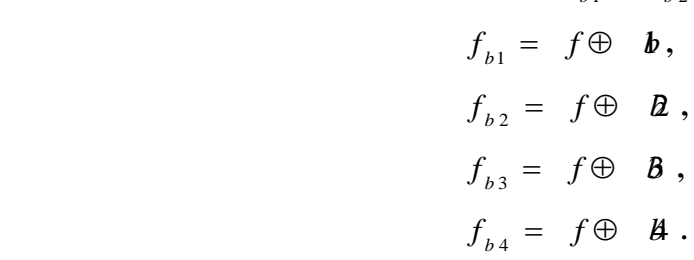

(2) Construct the vein image by using the weighted method, and the weighted formula is as follows.

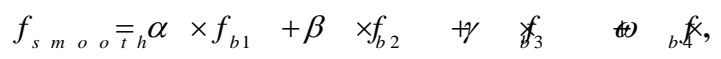

In formula-(5) $\gamma \alpha, \beta, \gamma, \omega$ are the weighting coefficients, respectively standing for each sub-inage's weight in the constructed image. The values of four parameters $\alpha, \beta$, $\gamma$ need to meet the equation $\alpha+\beta+\gamma+\omega=1$, and $\alpha \geq 0, \beta \geq 0, \gamma \geq 0$ and $\omega \geq 0$.

\subsection{Extraction of Light and Dark Area Information from Image}

Multiscale top-hat transformation is to use structural elements with multiple same shapes and different scales, so as to carry out top-hat transformation [62]. Design a group of structural elements with the same shape and increased scale: $b_{0}, b_{1}, \mathrm{~K}, b_{t},(t=1,2, \mathrm{~K})$, where $b_{0}$ is the unit structural element of scale 1 , $b_{i}=b_{0} \oplus b_{0} \oplus \mathrm{L} \oplus b_{0} \quad$ ( $b_{0} \quad$ is dilated by itself for $i$ times). Carry out top-hat conversion through structural elements with different scales, so as to extract the detail feature from images with different scales.

Multi-scale white top-hat conversion formula: 


$$
W T H_{i}=f-f \circ b_{i},
$$

$W H_{i}$ stands for white top-hat conversion by using $b_{i}$ in the image $f$ in the $i$ scale, including the light area details with scale less than $b_{i}$ in the image $f$.

Multi-scale black top-hat conversion formula:

$$
B T H_{i}=f \bullet b_{i}-f,
$$

$B T H_{i}$ stands for black top-hat conversion by using $b_{i}$ in the image $f$ in the $i$ scale, and including the dark area details with scale less than $b_{i}$ in the image $f$.

To extract the light and dark areas details with different scales, respectively use $W T H_{i(i-1)}$ and $B T H_{i(i-1)}$ to represent the residual image, which are defined as follows.

$$
W T H_{i+1}=W T_{i} H \quad W_{i-1},
$$

$W T H_{i(i-1)}$ includes the light area details in the image $f$ with the scale greater than $b_{i-1}$ and less than $b_{i}$.

$$
B T H_{i(i-1)}=B T H_{i}-B T H_{i}
$$

$B T H_{i(i-1)}$ includes the dark area details in the image $f$ with the scale greater than $b_{i-1}$ and less than $b_{i}$.

The specific methods for extracting the light and dark area information from finger images are as follows:

(1) Extract light area information from the image:

$$
f_{W}=\sum_{i=0}^{m} W T H_{i} / \hat{m}+\sum_{i=1}^{m} W T H_{i(i-1)} /(m-1),
$$

In formula (10), $f_{W}$ stands for multi-scale white top-hat conversion results, including the light area feature from 0 to $\mathrm{m}$ scale in the finger vein image.

(2) Extract dark area information from the image:

$$
1 f_{B}^{n}=\sum_{i=0}^{n} W T H_{i} / n+\sum_{i=1}^{n} W T H_{i(i-1)} /(n-1),
$$

In førmula (11), $f_{B}$ stands for multi-scale black top-hat conversion results, including the light area feature from 0 to $n$ scale in the finger vein image.

\subsection{Get the Enhancement Image}

(1) Map $f, f_{W}$ and $f_{B}$ in the image to the interval $[0,1]$.

$$
\begin{gathered}
f_{L}(x, y)=f(x, / y), \\
f_{W L}(x, y)={ }_{W} f(x, / y) \quad, \\
f_{B L}(x, y)=f_{B}(x, y) / B L,
\end{gathered}
$$

In formulas (12) (14), $L, W L, B L$ represent the maximum value in images $f, f_{W}$, $f_{B}$, and $f_{L}, f_{W L}, f_{B L}$ respectively represent the mapped results.

(2) Use enhancement operators for nonlinear conversion towards $f_{L}$, and the 
converted result is represented by $f_{L}^{\prime}$.

$$
f_{L}^{\prime}(x, y)=G \quad f_{L} x(y,=)\left\{\begin{array}{l}
\left.2\left(f_{L}(x),{ }^{2}\right)\right), \quad \leq f_{L} \quad x \quad y \leq 0 k \\
1-2\left(4 f_{L} x\left(y,{ }^{2}\right)\right), k<f_{L} x \quad y \leq
\end{array}\right.
$$

In formula (3.21), $k$ represents the crossover point, $k \in[0,1]$. The value of $k$ will be discussed in the experimental part.

(3) Use formula (13) to get the difference image of finger vein image, which is represented by $f_{\text {sub }}$.

$$
f_{\text {sub }}(x, y)=f_{W L}(x, y)-f_{B L}(x, y),
$$

The value $f_{\text {sub }}(x, y)$ describes the gray value information at $(x, y)$ in the finger vein image $f$. The larger value the $f_{\text {sub }}(x, y)$ is, the greater possibility that the original image at $(x, y)$ in the original image $f$ is the background area.

(4) For finger vein image with low contras directly add $f_{\text {a }}$ with $f_{L}^{\prime}$, and the effects are unobvious. Therefore, it is essential to set an enhancement coefficient for $f_{\text {sub }}$, and the coefficient is represented by $s$.

$$
f_{e n}(x, y)=f_{L}^{\prime}(x, y)+\lesssim x f_{s u b}(x, y)
$$

In formula (14), $f_{e n}$ represents the image after enhancement. The value of $s$ will be discussed in the experimental part.

(5) After being enhanced through the steps (2) (4), some images might generate the issue of relatively concentrated gray distribution. Therefore, it is essential to conduct a gray stretch for images and adjust the gray originally concentrated from $\left[g_{\min }, g_{\max }\right]$ to $[0,255]$. The gray stretch method is as follows:

$$
f_{e n}(x, y)=\frac{f_{e n}(x, \sqrt{x})-g_{\text {min }}}{g_{\text {min }}} \times 255,
$$

In formula (15), $g_{\max }$ represents the maximum gray value in image $f_{e n}$ and $g_{\text {min }}$ represents the minimum gray vale in image $f_{e n}$.

\subsection{FyGM Algorithm Flow Process}

The algorithm is shown in below chart.

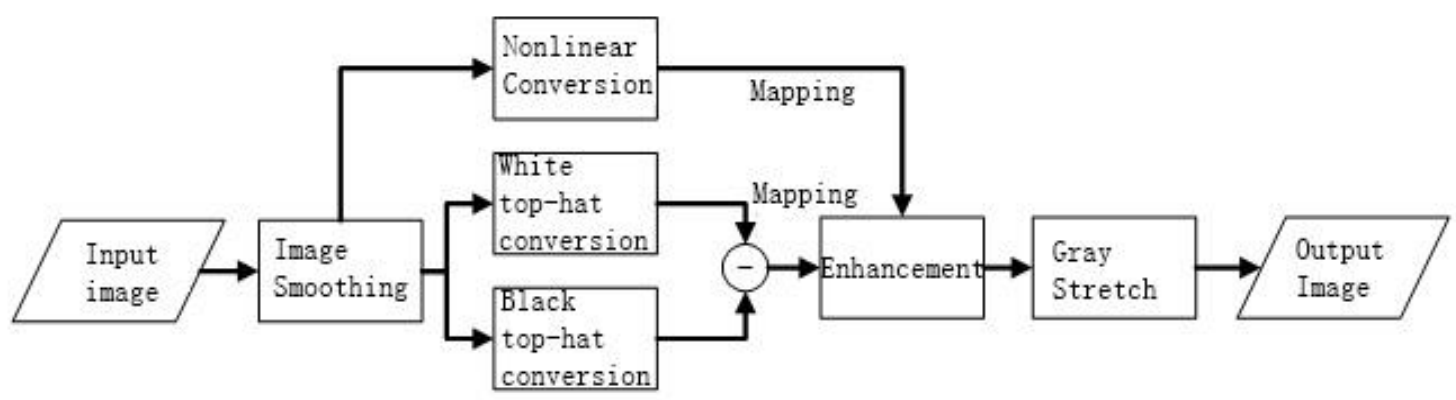

Figure 4. FVGM Algorithm Flow Chart 


\section{Algorithm Simulation}

\subsection{Selection of Weighted Parameter}

To describe the effects of structural elements' dilation operation in different directions, this paper calculates the Peak Signal to Noise Ratio (PSNR) of four sub-images to evaluate the four sub-images. Image resolution is an indicator measuring the image contour definition. The greater the value is, the more obvious the image's contour is, which is usually calculated by using the average grayscale gradient method (GMG). PSNR is an objective indicator to describe Peak Signal to Noise Ratio. The greater the value is, the more powerful the algorithm's denoising ability is. The calculation methods for GMG and PSNR are as follows:

$$
P S N R=10 \log _{10}\left(\frac{255^{2}}{M S E}\right),
$$

Where

$$
M S E=\sqrt{\frac{\sum_{x=1}^{M} \sum_{y=1}^{N}\left(f(x, y)-f^{\prime}(x, y)\right)^{2}}{M \times N}}
$$

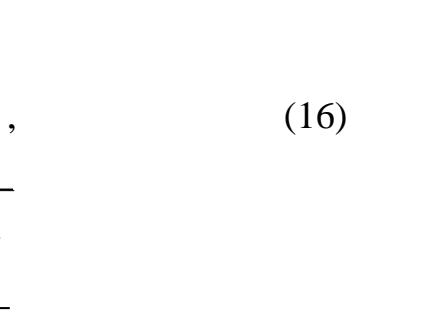

The calculation method of GMG is:

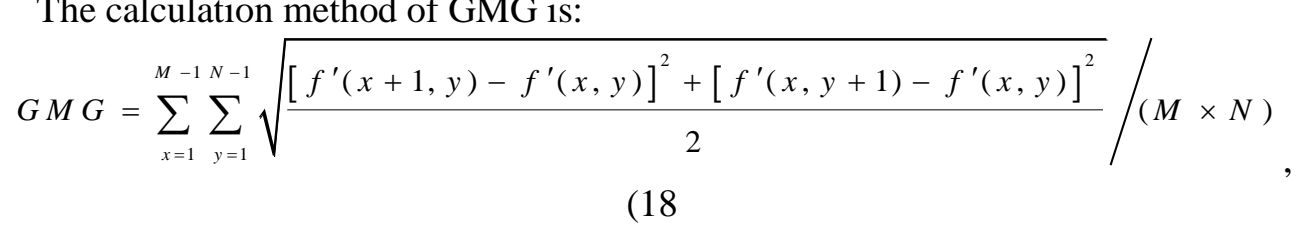

In formulas (17) ando(18), $f(x, y)$ represents the gray value of dilated image at $(x, y)$, $f(x, y)$ represents the gray value of original image at $(x, y), M$ and $N$ are the image's width and herght.

Figure 5(b)-(c) shows the results of four structural elements' respective dilation operation towards figure $5(\mathrm{a})$.

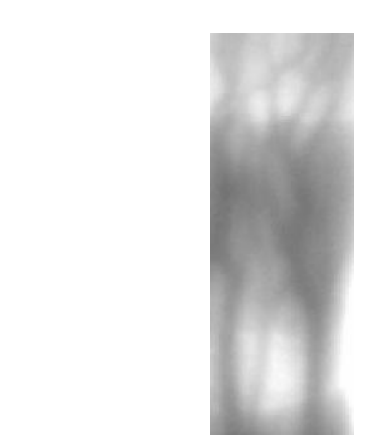

(a) $f$

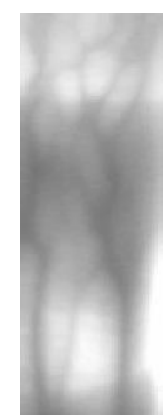

(b) $f_{b 1}$

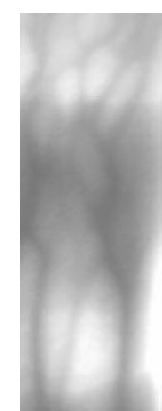

(c) $f_{b 2}$

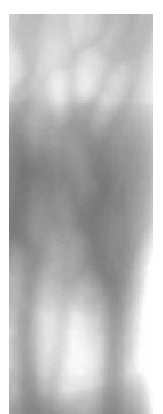

(d) $f_{b 3}$

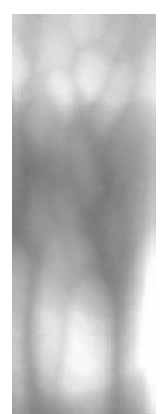

(e) $f_{b 4}$

Figure 5. Dilation Results

Table 1. Four Sub-images' GMG and PSNR Values

\begin{tabular}{ccccc}
\hline Image & $f_{b 1}$ & $f_{b 2}$ & $f_{b 3}$ & $f_{b 4}$ \\
\hline PSNR( dB $)$ & 33.4281 & 32.6156 & 37.9860 & 32.4151 \\
GMG & 11.2259 & 10.9765 & 9.7909 & 10.9201 \\
\hline
\end{tabular}


Table 1 shows four images' GMG and PSNR. According to Table 1, $f_{b 3}$ 's GMG value is lower than $f_{b 2}, f_{b 3}$ and $f_{b 4}$, whereas $f_{b 3}$ 's PSNR value is higher than the other three sub-images. This is because the intravenous lines in the original image is mainly distributed at $90^{\circ}$ and the adjacent direction. When using the structural elements at $90^{\circ}$ for dilation image, the image's PSNR value is relatively higher, which will lose the image definition. When using the structural elements at $0^{\circ}$ direction for dilation image, GMG value is higher than the other three images, but the PSNR value is less than $f_{b 3}$, indicating the structural elements at $0^{\circ}$ have better image edge protection effects yet with weaker denoising ability.

\subsection{Comparison with Other Three Smoothing Algorithm}

Figure 6 shows the effects of common image smoothing algorithms as well as the comparison design sketch with the algorithm in this chapter. Figure 6 (a) is the original finger vein image, Figure 6(b) shows the resultafter using a $5 \times 5$ square template neighborhood average filtering, Figure 6(c) shows the result of $4 \times 4$ square template median filtering, and Fig 6(d) shows the result after using a Gaussian template for low-pass filtering. These three methods have played a rote in smoothing the image and noise suppression. However, the finger veln edge becomes more blurred after filtering. Figure 6(e) shows the filtering results after using the morphology filter. Table 2 shows these four algorithms' PSNR and GMIG, values.

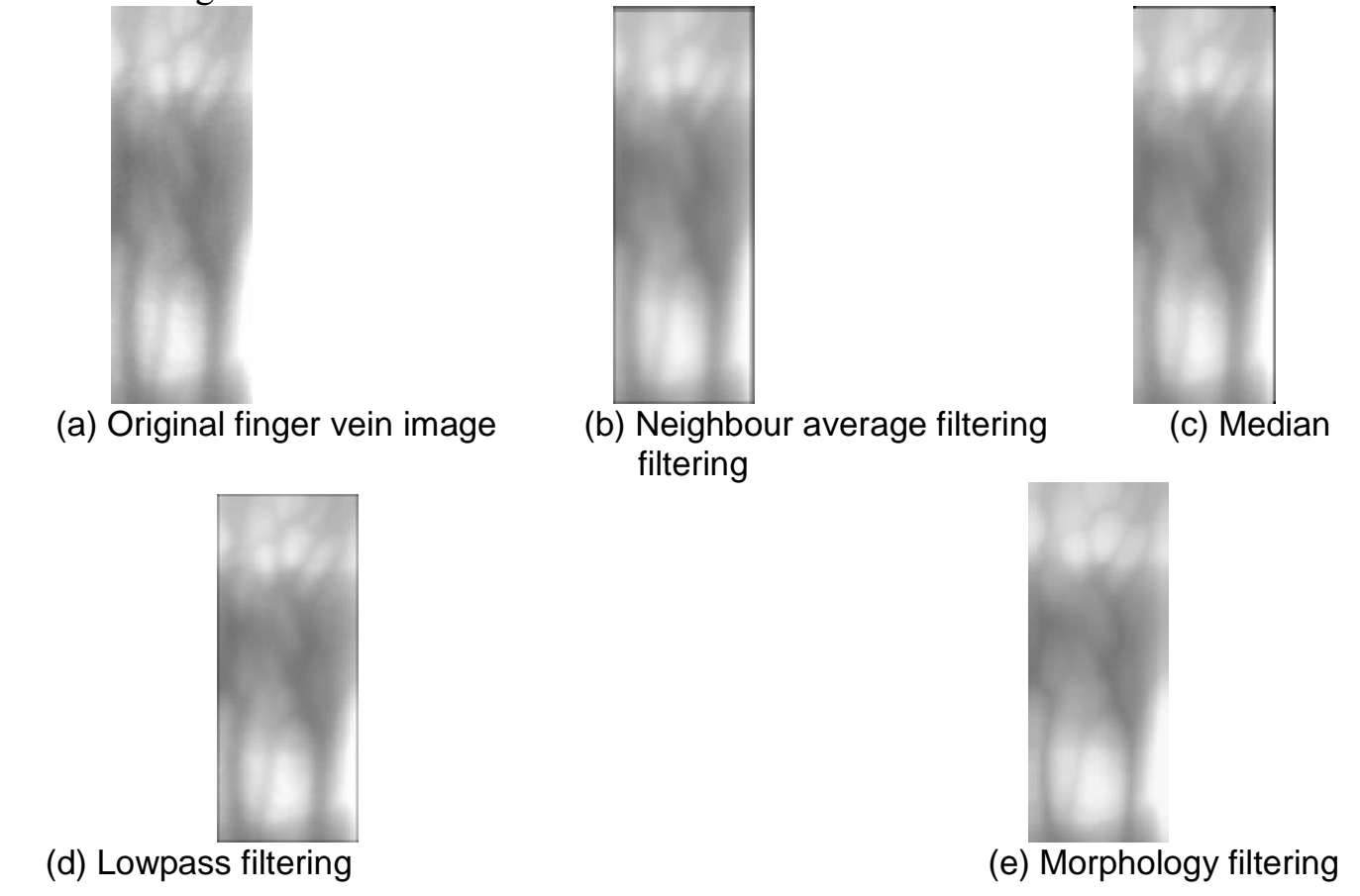

Figure 6. Comparison of Four Smoothing Algorithms

Table 2 shows that the morphology filtering algorithm's PSNR value and GMG value are higher than the other three algorithms, indicating the image edge information can be better protected when smoothing the image by using the filter constructed through morphological methods. 
Table 2. Four Algorithms' Comparison of PSNR and GMG Values

\begin{tabular}{ccccc}
\hline Algorithm & $\begin{array}{c}\text { Neighbour } \\
\text { average filtering }\end{array}$ & Median filtering & $\begin{array}{c}\text { Lowpass } \\
\text { filtering }\end{array}$ & $\begin{array}{c}\text { Morphology } \\
\text { filtering }\end{array}$ \\
\hline PSNR (dB) & 22.9150 & 23.5761 & 28.6881 & 34.3967 \\
GMG & 9.9526 & 9.5622 & 9.4552 & 10.1001 \\
\hline
\end{tabular}

\section{Conclusion}

This paper puts forward a Finger Vein Image Enhancement Algorithm Based on the Grayscale Morphology (FVGM). The algorithm first uses a group of structural elements with four directions for respective dilation operation towards images, and obtains the finger vein image after smoothing by the weighted method. After smoothing the image, the algorithm adopts the multiscale top-hat conversion to extract the finger vein inage's light and dark information on different scales, and get the enhancement image by using the fuzzy enhancement, gray stretch and other methods. Simulation results show that FVGM algorithm can effectively improve the definition of finger vein image's intravenous lines.

\section{Acknowledgement}

Science Technology Bureau of Shaoxing (2013B0722).

\section{References}

[1] Jain, A.K., A. Ross, S. Prabhakar. An Introduction to Biometric Recognition [J]. IEEE Transactions on Circults and Systems for Video Technology, 2004. 14(1):4-20.

[2] Ross, A. A., K. Nandakumar, A.K. Jain. Handbook of Multi-biometrics [M]. Springer-Verlag, 2007?

[3] Lu Guangming, Research on Key Technologies and Algorithms of Palm Print Identification System [D] Harbin: Harbin Institute of Technology, 2005.

[4] Sun Dongmei, Q1y Zhengding. Overview of Biometric Identification Technology [J]. Journal of Electronics, 2001,29 12):1744-1748.

[5] Qiu Xiaoming, et al. Personalized Fusion Method Based on Finger Vein and Finger Contour [J]. Computer Research and Development, 2013,50(9):1914-1923

[6] Yang Ying, et al Finger Vein Recognition Method after Fusing Local Features with Global Features [1]. Computer Engineering and Applications, 2012.48(12):158-162

[7] Liu Jianke. Low Contrast Finger Vein Enhancement for Uneven Illumination [J]. Microelectronics and Computer, 2012,29(12):91-93.

[8] Wang Kejun. Finger Vein Recognition Method based on Relative Distance [J]. Computer Science, 2011,38(9):257-259.

[9] Li Liubo. Simulation Research on Improved Finger Vein Image Recognition Algorithm [J]. Computer Simulation, 2011,28(3):310-312.

[10] Yu Chengbo, et al. Research on Finger Vein Feature Indirect Extraction of Image Residuals [J]. Computer Engineering and Applications, 2010,46(28):167-174

\section{Author}

Chen Xuan (1979-), male, master, lecturer, research direction: information security, wireless sensor and cloud computing. Zhang Jun (1989-), male, graduate, lecturer, research direction: information security and biometric identification. You Lin (1966-), male, Ph.D., professor, research direction: information security. Wang Changliang (1973-), male, master, associate professor, research direction: computer network and algorithm design, Xu Fangheng (1980.9-) male, master, instructor, research direction: database and information system, information security. 
International Journal of Multimedia and Ubiquitous Engineering

Vol.9, No.8 (2014)

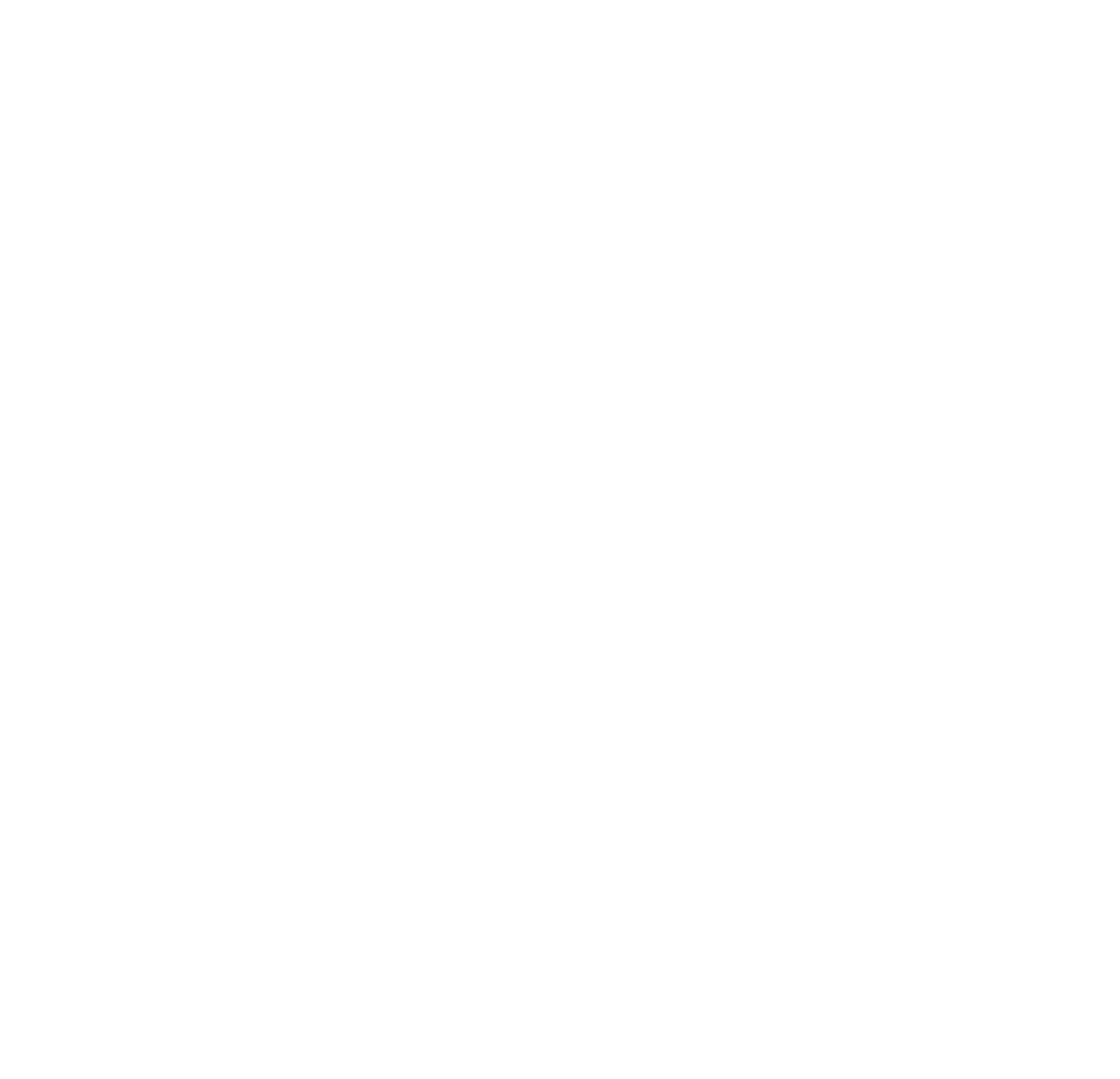

